\title{
Tumore bruno della mandibola in un paziente con iperparatiroidismo primitivo
}

\author{
R. Severino ${ }^{1}$, V. Ramundo ${ }^{1}$, L. Vuolo ${ }^{1}$, C. Di Somma ${ }^{1}$, G. Lombardi ${ }^{1}$, A. Colao ${ }^{1}$, \\ S. Spiezia ${ }^{2}$, A. Faggiano ${ }^{1}$
}

${ }^{1}$ Dipartimento di Endocrinologia e Oncologia Molecolare e Clinica, Università "Federico II" di Napoli, Napoli ${ }^{2}$ Unità Operativa di Chirurgia Ecoguidata e Patologie del Collo, P.O. Incurabili, ASL Napoli 1 Centro, Napoli

\section{Caso clinico}

Nel febbraio 2010 un paziente di 77 anni giunge per un controllo endocrinologico presso il Dipartimento di Endocrinologia del Policlinico Universitario "Federico II" di Napoli, con una diagnosi di tumore bruno dell'osso mascellare effettuata su prelievo bioptico, iperparatiroidismo, ipercalcemia e insufficienza renale cronica.

Nel luglio 2009 il paziente praticava un ricovero presso il reparto di Odontostomatologia per un approfondimento diagnostico relativo ad una tumefazione in regione mascellare. All'anamnesi il paziente riferiva pregressa storia di nefrolitiasi, con ripetuti episodi di coliche renali. Una TC del massiccio facciale mostrava un'area osteolitica a carico della metà sinistra dell'osso alveolare superiore, in associazione ad altri difetti osteolitici a carico della mandibola. All'esame bioptico, si poneva la diagnosi istologica di tumore bruno.

$\mathrm{Si}$ effettuava una valutazione ematochimica che mostrava una condizione di insufficienza renale con concentrazioni sieriche di creatinina di $2,3 \mathrm{mg} / \mathrm{dL}$, cui si associava un quadro suggestivo di iperparatiroidismo (calcio: 10,3 mg/dL; fosforemia: $3 \mathrm{mg} / \mathrm{dL}$; PTH: $1120 \mathrm{pg} / \mathrm{mL}$ ). Un'ecografia renale evidenziava la perdita di differenziazione cortico-midollare e la presenza di calcoli di 15 e $12 \mathrm{~mm}$ rispettivamente a destra e a sinistra. Una scintigrafia delle paratiroidi mostrava la presenza di tessuto iperfunzionante in corrispondenza del terzo inferiore del lobo sinistro della tiroide.

Veniva pertanto ricoverato in Endocrinologia. All'ammissione in reparto il paziente riferiva dolori ossei diffusi e una frattura recente dell'omero sinistro che alla radiografia appariva non completamente consolidata.

Gli esami ematochimici mostravano iperparatiroidismo primitivo, anemia ipocromica ed un modico peggioramento della funzionalità renale (creatininemia $2,3 \mathrm{mg} /$
dL, 3 mesi dalla prima valutazione effettuata in Odontostomatologia) (Tab. I).

Sulla base della storia anamnestica (nefrolitiasi da circa 30 anni prima), delle elevate concentrazioni di PTH ( $>1500 \mathrm{pg} / \mathrm{mL}$ ) e del quadro eco-color-Doppler, che mostrava un adenoma paratiroideo di $4,5 \mathrm{~cm}$ alla base del lobo sinistro della tiroide (Fig. 1), si escludeva la diagnosi di iperparatiroidismo terziario (autonomizzazione funzionale in corso di pregresso iperparatiroidismo secondario) e si poneva la diagnosi di iperparatiroidismo

TABELLA I - PARAMETRI EMATOCHIMICI ALLA DIAGNOSI

\begin{tabular}{|l|c|}
\hline Creatinina & $2,3 \mathrm{mg} / \mathrm{dL} \uparrow$ \\
\hline Urea & $103 \mathrm{mg} / \mathrm{dL} \uparrow$ \\
\hline Microalbuminuria & $66,5 \mathrm{mg} / \mathrm{L} \uparrow$ \\
\hline Potassiemia & $6,3 \mathrm{mmol} / \mathrm{L} \uparrow$ \\
\hline RBC & $3,08 \mathrm{mln} / \mathrm{mcL} \downarrow$ \\
\hline Hb & $8,3 \mathrm{~g} / \mathrm{dL} \downarrow$ \\
\hline HCT & $26,00 \% \downarrow$ \\
\hline MCV & $85 \mathrm{fl}$ \\
\hline MCH & $26,9 \mathrm{pg}$ \\
\hline Sideremia & $36 \mathrm{mcg} / \mathrm{dL} \downarrow$ \\
\hline Ferritinemia & $139 \mathrm{ng} / \mathrm{mL}$ \\
\hline Calcemia & $11,7 \mathrm{mg} / \mathrm{dL} \uparrow$ \\
\hline Fosforemia & $2,8 \mathrm{mg} / \mathrm{dL} \downarrow$ \\
\hline PTH & $1687 \mathrm{pg} / \mathrm{mL} \uparrow$ \\
\hline Calciuria & $50 \mathrm{mg} / 24 \mathrm{~h}$ \\
\hline Fosfaturia & $550 \mathrm{mg} / 24 \mathrm{~h}$ \\
\hline
\end{tabular}




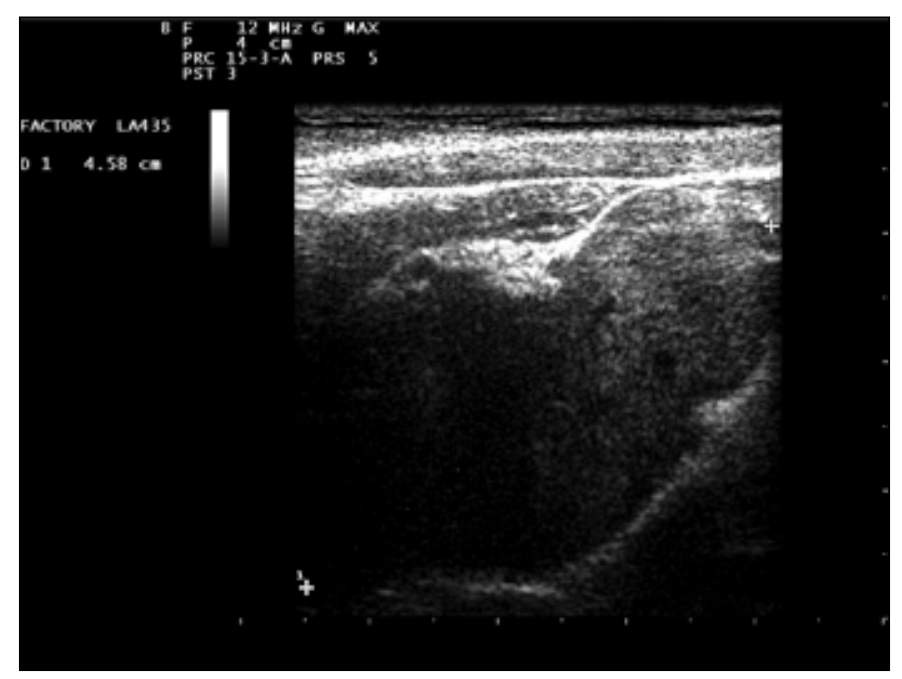

Fig. 1 - Ecografia della tiroide: area ipoecogena ovalare di $45 \times 38 \mathrm{~mm}$ da riferire a paratiroide iperplastica alla base del lobo sinistro.

primitivo complicato da nefrolitiasi cronica e conseguente insufficienza renale.

Si cominciava pertanto terapia con cinacalcet, eritropoietina, furosemide, bicarbonato di sodio e dieta iposodica. In considerazione delle notevoli dimensioni dell'adenoma paratiroideo e degli elevatissimi livelli di PTH si decideva inoltre di inviare il paziente all'intervento chirurgico di adenomectomia paratiroidea. Nell'attesa dell'intervento è stata iniziata terapia con cinacalcet che ha indotto un iniziale decremento dei livelli di PTH e calcemia (Figg. 2 e 3 ).

\section{Discussione}

Liperparatiroidismo è un disordine endocrino caratterizzato da una eccessiva produzione di PTH da parte delle paratiroidi. Questa condizione può dipendere sia da un'autonomia funzionale delle ghiandole (iperparatiroidismo primario) in presenza di iperplasia, adenoma o carcinoma delle paratiroidi, sia da un'alterazione del metabolismo calcio-fosforo con secondaria iperattivazione della funzione paratiroidea (iperaparatiroidismo secondario), come si verifica nell'insufficienza renale cronica, ipovitaminosi D ecc.

L’iperparatiroidismo primario è il terzo più comune disordine endocrino $(1,2)$. La diagnosi si basa sul dosaggio contestuale della calcemia, della fosforemia e del PTH. La diagnosi di iperparatiroidismo primario è sempre più frequente e precoce negli ultimi anni grazie alla diffusa misurazione della calcemia tra le indagini ematochimi-

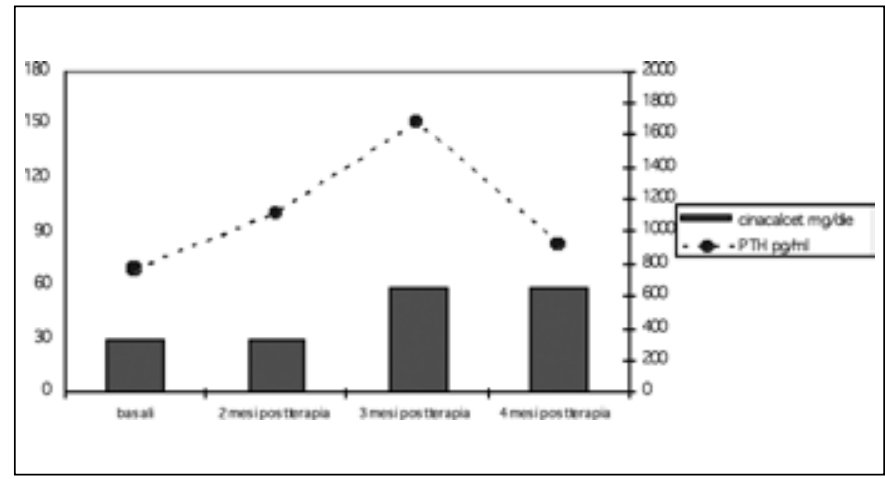

Fig. 2 - Concentrazioni sieriche di PTH prima e dopo terapia con cinacalcet.

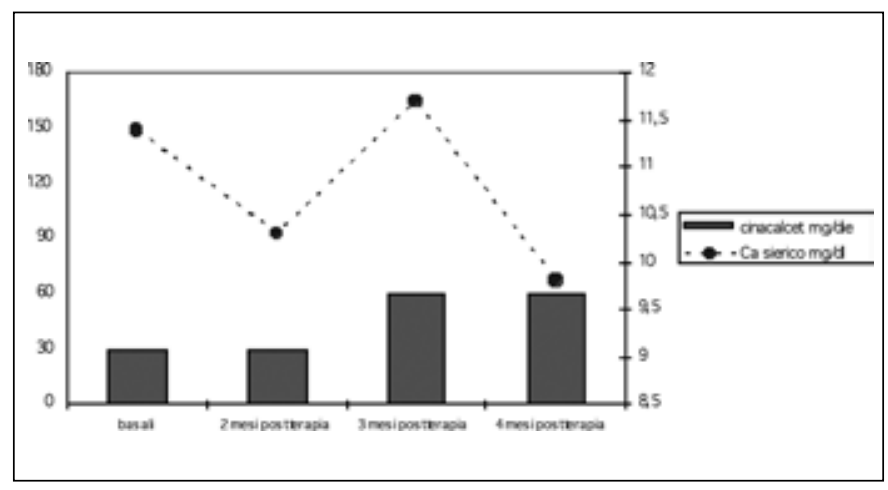

Fig. 3 - Concentrazioni sieriche di calcio prima e dopo terapia con cinacalcet.

che di routine: ciò porta ad evidenziare l'iperparatiroidismo primario in fase iniziale in pazienti asintomatici o paucisintomatici.

Meno frequentemente l'iperparatiroidismo primario si manifesta clinicamente con sintomi correlati agli elevati livelli di calcio sierico e urinario, tra cui ci sono calcoli renali, osteoporosi, disturbi gastrointestinali e a carico del sistema neuromuscolare. Molto più raro (non più del 3-4 \% dei casi di iperparatiroidismo primitivo secondo le stime che si riferiscono agli ultimi 20 anni) è lo sviluppo di un tumore bruno $(2,3)$. Esso rappresenta lo stadio terminale del processo di rimodellamento dell'osso promosso dagli elevati livelli di PTH, che causano iperattivazione degli osteoclasti e iperproliferazione dei fibroblasti (4).

Il caso clinico descritto si riferisce ad una condizione di iperparatiroidismo primitivo a lungo misconosciuta $\mathrm{e}$ conseguente prolungata esposizione dell'osso e dei reni ad elevati valori di PTH. Si è instaurato pertanto un quadro di compromissione renale e successivamente lo sviluppo della lesione mandibolare prima che si giungesse 
alla diagnosi di iperparatiroidismo primario. Al momento della diagnosi il paziente appariva paucisintomatico nonostante le severa compromissione ossea e renale. Tale esiguità dei sintomi è da riferire all'età avanzata del paziente e alla progressione molto graduale della patologia. La particolarità di questo caso è che la diagnosi di iperparatiroidismo primario è stata fatta sulla base dei sintomi e segni del tumore bruno (dolore, tumefazione con deformazione dei lineamenti) che rappresenta lo stadio finale della malattia $(3,5)$.

Dal punto di vista terapeutico la terapia chirurgica si è resa necessaria per la marcata attività proliferativa e secretoria dell'adenoma paratiroideo. Tuttavia, la terapia te controllare l'ipercalcemia e l'ipercalciuria stabilizzando il quadro clinico ed ematochimico del paziente.

Il cinacalcet è un calcio mimetico che agisce legando il recettore per il calcio posto sulle cellule delle paratiroidi, principale regolatore della secrezione di PTH. Aumentando la sensibilità al calcio extracellulare di tali recettori, il cinacalcet riduce la secrezione di PTH da parte delle cellule paratiroidee $(6,7)$.

La terapia con calcio mimetici è stata in passato riservata ai casi di iperparatiroidismo secondario. La terapia definitiva dell'iperparatiroidismo primario è la paratiroidectomia. Per pazienti che non rispondono ai criteri di operabilità, in attesa dell'intervento chirurgico o nei quali la condizione di iperparatiroidismo è refrattaria alla terapia chirurgica, viene presa in considerazione la terapia medica con calcio mimetici. E stato infatti dimostrato che il cinacalcet normalizza i livelli di calcemia contestualmente ad una riduzione dei livelli di PTH $(6,8)$. medica con cinacalcet ha mostrato di poter efficacemen-

\section{Riassunto}

Gli Autori riportano un caso clinico in cui la diagnosi di tumore bruno dell'osso mascellare ha condotto alla definizione del processo patologico primitivo causato da un iperparatiroidismo sostenuto da adenoma primitivo della paratiroide. Il paziente, portatore di insufficienza renale cronica e nefrolitiasi ha eseguito biopsia e TC del mascellare, con diagnosi di tumore bruno associato ad altre aree di osteolisi a carico dell'osso mandibolare. Il profilo ematochimico ha rivelato una ipercalcemia con ipofosforemia associata a aumento del dosaggio sierico del paratormone, pertanto è stata eseguita una scintigrafia delle paratiroidi che ha mostrato la presenza di tessuto iperfunzionante in corrispondenza del terzo inferiore del lobo sinistro della tiroide. Il quadro eco-color-Doppler, ha confermato la presenza di un adenoma paratiroideo di $4,5 \mathrm{~cm}$ alla base del lobo sinistro della tiroide. Il paziente è stato sottoposto a terapia con cinacalcet in attesa dell'intervento chirurgico.

Parole chiave: Iperparatiroidismo primitivo, Tumore bruno, PTH, Ipercalcemia, Cinacalcet

\section{TEST di VERIFICA - 7}

\section{1) L’iperparatiroidismo primitivo è più spesso causato da:}

a) Adenoma paratiroideo singolo

b) Carcinoma paratiroideo

c) Iperplasia diffusa delle paratiroidi

d) Nessuna delle precedenti

\section{2) Il cinacalcet è un calciominetico che, legandosi ai recettori del calcio, determina:}

a) Aumento della calcemia sierica

b) Aumento del PTH e riduzione della calcemia

c) Riduzione del PTH e riduzione della calcemia

d) Nessuna delle precedenti

\section{3) Il cinacalcet è indicato nel trattamento di:}

\section{Indirizzo degli Autori:}

Antongiulio Faggiano, MD

Dipartimento di Endocrinologia e

Oncologia Molecolare e Clinica

Università "Federico II" di Napoli

Via Sergio Pansini 5

80131 Napoli

afaggian@unina.it a) Iperparatiroidismo secondario ad IRC (insufficienza renale cronica)

b) Iperparatiroidismo primitivo non operabile

c) Iperparatiroidismo da carcinoma paratiroideo

d) Tutte le precedenti

Le risposte corrette alle domande sono a pag. 24 


\section{Bibliografia}

1. Fraser WD. Hyperparathyroidism. Lancet 2009; 11; 374 (9684): 145-58.

2. Błazejewska A, Flisiński M, Pypkowski J, Manitius J. Patient with chronic kidney disease and primary or tertiary hyperparathyroidism-case report. Merkur Lekarski 2009; 27(162): 484-7.

3. Di Daniele N, Condò S, Ferrannini M, Bertoli M, Rovella V, Di Renzo L, De Lorenzo A. Brown tumour in a patient with secondary hyperparathyroidism resistant to medical therapy: case report on successful treatment after subtotal parathyroidectomy. Int J Endocrinol 2009; 2009: 827652.

4. Sutbeyaz Y, Yoruk O, Bilen H, Gursan N. Primary hyper- parathyroidism presenting as a palatal and mandibular brown tumor. J Craniofac Surg 2009; 20 (6): 2101-4.

5. Tarello F, Ottone S, De Gioanni PP, Berrone S. Brown tumor of the jaws. Minerva Stomatol 1996; 45(10): 465-70.

6. Shoback DM, Bilezikian JP, Turner SA, McCary LC, Guo MD, Peacock M. The calcimimetic cinacalcet normalizes serum calcium in subjects with primary hyperparathyroidism. J Clin Endocrinol Metab 2003; 88(12): 5644-9.

7. Wüthrich RP, Martin D, Bilezikian JP. The role of calcimimetics in the treatment of hyperparathyroidism. Eur J Clin Invest. 2007; 37(12): 915-22.

8. Dillon ML, Frazee LA. Cinacalcet for the Treatment of Primary Hyperparathyroidism. Am J Ther 2010 Mar 11. [Epub ahead of print] 